\title{
OPEN- AND CLOSED-LOOP EQUILIBRIUM CONTROL OF TROPHIC CHAINS
}

\author{
M. Gámez ${ }^{\text {a }}$, I. López ${ }^{a}$, A. Shamandy \\ a Department of Statistics and Applied Mathematics, University of Almería, \\ La Cañada de San Urbano,04120 Almería,Spain.(mgamez@ual.es, \\ milopez@ual.es) \\ ${ }^{\mathrm{b}}$ Department of Mathematics, Faculty of Science, University of Mansoura, Egypt. \\ (shamandy16@hotmail.com)
}

\begin{abstract}
If a nearly natural population system is deviated from its equilibrium, an important task of conservation ecology may be to control it back into equilibrium. In the paper a trophic chain is considered, and control systems are obtained by changing certain model parameters into control variables. For the equilibrium control two approaches are proposed. First, for a fixed time interval, local controllability into equilibrium is proved, and applying tools of optimal control, it is also shown how an appropriate open-loop control can be determined that actually controls the system into the equilibrium in given time. Another considered problem is to control the system to a new desired equilibrium. The problem is solved by the construction of a closed-loop control which asymptotically steers the trophic chain into this new equilibrium. In this way, actually, a controlled regime shift is realized.
\end{abstract}

Keywords: trophic chains, controllability, optimal control, equilibrium control

\section{Introduction}

The concept of control of a trophic chain is used in different senses in the literature. A possible classification is: internal natural control, external natural control, external control by management. (For an overview of the different types of ecosystem control see Fath, 2004). Our study joins the research line concerning external, human control of trophic chains.

The human influence on ecosystems, in particular on population systems, is an important issue in conservation ecology. Moreover, sustainability of economic and social development in a broader sense also involves conservation aspects of ecology. On the one hand, ecosystems are often exposed to a strong human intervention, such as economic activity, wildlife management, fisheries or environmental pollution. On the

*Corresponding author. Fax: +34-950015167; Phone: +34-950015667

E-mail address: mgamez@ual.es (M. Gámez) 
1 other hand, if the human activity breaks the equilibrium of the population system in question, we can try to control it back to the previous or a new equilibrium.

These problems make it necessary to extend the traditional approach of theoretical biology focusing only on a biological object, to the study of systems consisting of a biological object and man that monitors or/and controls the biological object. This, in dynamic situation, i.e. in case of a long-term human intervention, typically requires the approach of mathematical systems theory (in frequently used terms, state-space modelling), see Kalman et al. (1969) for the basic results of this theory, and Chen et al. (2004) for a recent reference. This methodology offers solutions not only to controlling but also to monitoring (i.e. observation) problems of population systems. While by now, mathematical systems theory became quite familiar to system engineers, observability and controllability analysis of dynamic models in population biology is relatively new. The results on controllability and observability in frequency-dependent population genetics models are mostly based on the sufficient conditions obtained in Varga (1989), (1990) and (1992), for the control and observation of systems with invariant manifold. For the applications of these theorems see e.g. Kósa and Varga (1996), Scarelli and Varga (2002), López et al. (2004) and Varga (2008a).

For the control and monitoring problems of density-dependent population systems, the corresponding mathematical tools can be found in Lee and Markus (1971); conditions for controllability and observability problems for different Lotka-Volterra type systems have been obtained e.g. in Varga et al. (2003), Gámez et al. (2008), López et al. (2007). A recent general overview of the different applications of mathematical systems theory in population biology is Varga (2008b).

In the present paper ecological systems of non-Lotka-Volterra type will be considered, that form a trophic chain of type resource - producer - primary consumer, see e.g. Svirezhev and Logofet (1983), Yodzis (1989). Stability and observability results for such systems have been obtained in Shamandy (2005). We note that the monitoring of a somewhat different, four-level ecological interaction chain of type resource producer - primary user - secondary consumer has been studied, applying the mathematical results on verticum type systems, published in Molnár (1987), (1988a-e), (1989), (1993), Molnár and Szigeti (1994).

In Section 2, from Shamandy (2005), the model setup and basic conditions for the existence and stability of an equilibrium of the system are shortly recalled. Section 3 
1 and 4 is the main body of the paper. In Section 3 we prove the trophic chain is locally controllable into the equilibrium in given time. We also show how to calculate a corresponding open-loop control, applying the toolbox developed for MatLab in Banga, et al. (2005) and Hirmajer et al. (2009). In Section 4, based on results of Rafikov et al. (2008), we construct a closed-loop (actually a linear feedback) control that steers the system into a desired equilibrium. Section 5 is dedicated to the discussion of our results. Finally, in the Appendix we recall some basic concepts and results applied in the present paper.

\section{Description of the dynamic model}

For the presentation of our approach we consider a relatively simple food web, a trophic chain involving a resource, a plant and a herbivorous animal. In this section, from Shamandy (2005) we recall the dynamic model of a trophic chain of this type, see also Svirezhev and Logofet (1983), Jorgensen and Svirezhev (2004). For further details on trophic chains (and general food webs) we refer the reader to Yodzis (1989).

The considered model describes how a resource moves through a trophic chain. A typical terrestrial trophic chain consists of the following components:

resource, the $0^{\text {th }}$ trophic level (solar energy or inorganic nutrient),

which is incorporated by

a plant population, the $1^{\text {st }}$ trophic level (producer), which transfers it to

a herbivorous animal population, the $2^{\text {nd }}$ trophic level (primary consumer).

We note that for a similar study a longer trophic chain can also be considered, where the herbivore can be consumed by a predator population, the $3^{\text {rd }}$ trophic level (secondary consumer), which can be followed by top predator population (tertiary consumers). In the present paper, for technical simplicity, only trophic chains of the type resource producer - primary consumer will be studied. According to the possible types of $0^{\text {th }}$ level (energy or nutrient), two types of trophic chains will be considered: open chains (without recycling) and closed chains (with recycling). At the $0^{\text {th }}$ trophic level, resource will be the common term for energy and nutrient.

Let us denote by $x_{0}$ the time-varying quantity of "free" resource present in the system, $x_{1}$ and $x_{2}$, in function of time, the biomass (or density) of the producer (species 1) and the primary consumer (species 2), respectively. Let $Q$ be the resource supply considered constant in the model. Let $\alpha_{0} x_{0}$ be the velocity at which a unit biomass of 
1 species 1 consumes the resource, and it is assumed that this consumption increases the 2 biomass of this species at rate $k_{1}$. A unit biomass of species 2 consumes the biomass of 3 species 1 at velocity $\alpha_{1} x_{1}$, converting it into its own biomass at rate $k_{2}$. Both the plant and the animal populations are supposed to decrease exponentially in the absence of the resource and the other species, with respective rates of decrease (Malthus parameters) $m_{1}$ and $m_{2}$.

Recycling can also be included in the model: In a closed system the dead individuals of species 1 and 2 are recycled into free nutrient at respective rates $0<\beta_{1}<1$ and $0<\beta_{2}<1$, while for an open system (where there is no natural recycling) $\beta_{1}=0, \beta_{2}=0$ holds. (If only one of the $\beta-\mathrm{s}$ is positive, the system is called partially closed.) Then with model parameters

$$
\left.Q, \alpha_{0}, \alpha_{1}, m_{1}, m_{2}>0 ; k_{1}, k_{2} \in\right] 0,1\left[; \beta_{1}, \beta_{2} \in[0,1[,\right.
$$

the dynamic model for the trophic chain can be set up as follows:

$$
\begin{aligned}
& \dot{x}_{0}=Q-\alpha_{0} x_{0} x_{1}+\beta_{1} m_{1} x_{1}+\beta_{2} m_{2} x_{2} \\
& \dot{x}_{1}=x_{1}\left(-m_{1}+k_{1} \alpha_{0} x_{0}-\alpha_{1} x_{2}\right) \\
& \dot{x}_{2}=x_{2}\left(-m_{2}+k_{2} \alpha_{1} x_{1}\right)
\end{aligned}
$$

Let function $f$ be defined in terms of the right-hand side of this system:

$$
f: \mathbf{R}^{3} \rightarrow \mathbf{R}^{3}, f(x)=f\left(x_{0}, x_{1}, x_{2}\right):=\left[\begin{array}{c}
Q-\alpha_{0} x_{0} x_{1}+\beta_{1} m_{1} x_{1}+\beta_{2} m_{2} x_{2} \\
x_{1}\left(-m_{1}+k_{1} \alpha_{0} x_{0}-\alpha_{1} x_{2}\right) \\
x_{2}\left(-m_{2}+k_{2} \alpha_{1} x_{1}\right)
\end{array}\right]
$$

In Shamandy (2005), a necessary and sufficient condition has been obtained for the coexistence of the population system. The latter means that there exists a non-trivial ecological equilibrium $x^{*}$ of dynamic system (2.2)-(2.4), where all components are present: system (2.2)-(2.4) has a unique equilibrium $x^{*}=\left(x_{0}^{*}, x_{1}^{*}, x_{2}^{*}\right)>0$ if and only if the resource supply is high enough, i.e.

$$
Q>Q_{2}:=\frac{m_{1} m_{2}}{\alpha_{1} k_{1} k_{2}}-\frac{\beta_{1} m_{1} m_{2}}{\alpha_{1} k_{2}},
$$

and then the respective equilibrium values for the resource, plant and herbivore are 


$$
x_{0}^{*}=\frac{-\alpha_{1} Q-\frac{\beta_{1} m_{1} m_{2}}{k_{2}}+\beta_{2} m_{1} m_{2}}{-\frac{\alpha_{0} m_{2}}{k_{2}}+\alpha_{0} \beta_{2} k_{1} m_{2}},
$$

$$
x_{1}^{*}=\frac{m_{2}}{k_{2} \alpha_{1}}
$$

$$
x_{2}^{*}=\frac{-\alpha_{0} k_{1} Q-\frac{\alpha_{0} m_{1} m_{2}}{k_{2} \alpha_{1}}-\beta_{1} k_{1} m_{1} m_{2}}{-\frac{\alpha_{0} m_{2}}{k_{2}}+\alpha_{0} \beta_{2} k_{1} m_{2}} .
$$

4 Throughout the paper condition (2.5) will be supposed.

Remark 2.1. For $\beta_{1}>0$ the threshold $Q_{2}$ is lower than for $\beta_{1}=0$. Clearly, in the latter case the lack of recycling from species 1, a higher value of resource supply is necessary to produce the required positive equilibrium.

Remark 2.2. It can be shown that, under the same condition the stable coexistence is also guaranteed, or in mathematical terms, this equilibrium $x^{*}$ is asymptotically stable. In order to guarantee this stable coexistence $x^{*}$, we shall suppose throughout the paper that condition (2.5) holds.

Example 2.3. For an illustration, we consider system (2.2)-(2.4) with parameters $Q:=10 ; \alpha_{0}:=0.3 ; \alpha_{1}:=0.1 ; \beta_{1}:=0.2 ; \beta_{2}:=0.3 ; m_{1}:=0.1 ; m_{2}:=0.4 ; k_{1}:=0.5 ; k_{2}:=0.5$.

14 Checking condition (2.5), we get $Q>Q_{2}=1.44$, therefore in this case the considered system (2.2)-(2.4) has a positive equilibrium $x^{*}=(4.52,8,5.78)$ calculated from (2.6)-(2.8), which is asymptotically stable (see Figure 1). 


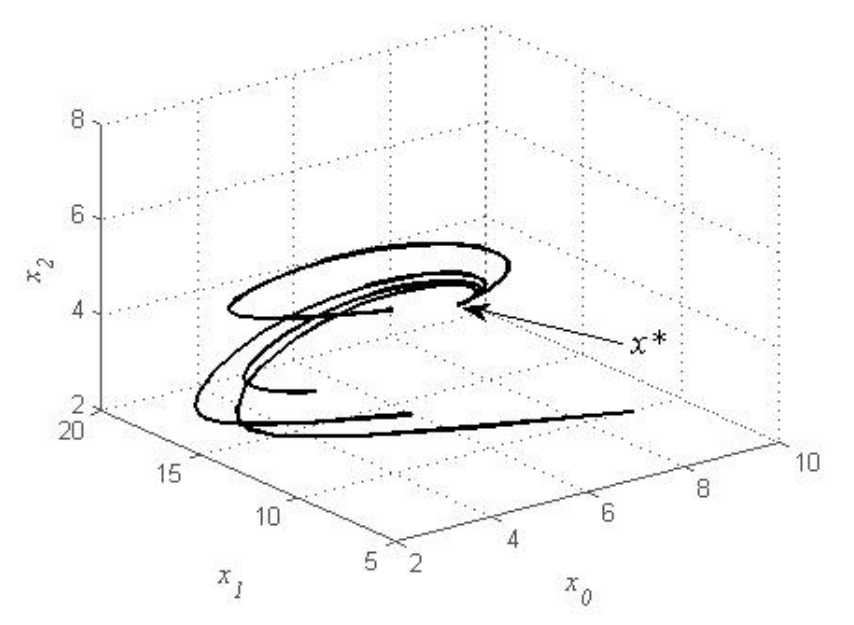

Figure 1. Some solutions of system (2.2)-(2.4)

1

\section{Open-loop control of the trophic chain into equilibrium in given time}

In this section we will deal with the following problem. Let us suppose that the system is deviated from its equilibrium, and we want to steer it back into equilibrium by changing certain model parameters into control variables. In mathematical terms this means that to the reference value of a model parameter (resource supply, recycling rate or Malthus parameter), a time-dependent control function is added. Open-loop control means that we want to determine in advance a control in function of time, such that the corresponding time-dependent state of the system reaches the original equilibrium in given time. (The closed-loop controls to be considered in the next section will depend on the current state of the system.)

\section{Case 1. Control of the resource supply}

Let us suppose first that the resource supply is controlled in function of time in the form $Q+u(t)$, considering control functions $u$ defined on a fixed interval $[0, \mathrm{~T}]$. Then our model (2.2)-(2.4) takes the form

$$
\begin{aligned}
& \dot{x}_{0}=Q+u(t)-\alpha_{0} x_{0} x_{1}+\beta_{1} m_{1} x_{1}+\beta_{2} m_{2} x_{2} \\
& \dot{x}_{1}=x_{1}\left(-m_{1}+k_{1} \alpha_{0} x_{0}-\alpha_{1} x_{2}\right) \\
& \dot{x}_{2}=x_{2}\left(-m_{2}+k_{2} \alpha_{1} x_{1}\right) .
\end{aligned}
$$

Then (3.1)- (3.3) can be considered as a control system, and in terms of the notation of the Appendix, with 


$$
F: \mathbf{R}^{4} \rightarrow \mathbf{R}^{3}, \quad F\left(x_{0}, x_{1}, x_{2}, u\right):=\left[\begin{array}{c}
Q+u-\alpha_{0} x_{0} x_{1}+\beta_{1} m_{1} x_{1}+\beta_{2} m_{2} x_{2} \\
x_{1}\left(-m_{1}+k_{1} \alpha_{0} x_{0}-\alpha_{1} x_{2}\right) \\
x_{2}\left(-m_{2}+k_{2} \alpha_{1} x_{1}\right)
\end{array}\right]
$$

2 Control system (3.1)-(3.3) takes the form

3

4

9 Since

$$
\dot{x}=F\left(x, u^{*}+u(t)\right)
$$

Obviously, to $u^{*}:=0$ and $u(t):=0 \quad(t \in[0, T])$, there corresponds the non-trivial ecological equilibrium $x^{*}$ of dynamic system (2.2)-(2.4).

Now we show that control system (3.4) is locally controllable to $x^{*}$ on $[0, T]$. For the application of Theorem A.2 of the Appendix, let us calculate the Jacobians

$$
A:=D_{1} F\left(x^{*}, 0\right)=\left[\begin{array}{ccc}
-\alpha_{0} x_{1}^{*} & -\alpha_{0} x_{0}^{*}+\beta_{1} m_{1} & \beta_{2} m_{2} \\
k_{1} \alpha_{0} x_{1}^{*} & 0 & -\alpha_{1} x_{1}^{*} \\
0 & k_{2} \alpha_{1} x_{2}^{*} & 0
\end{array}\right], B:=D_{2} F\left(x^{*}, 0\right)=\left[\begin{array}{l}
1 \\
0 \\
0
\end{array}\right] .
$$

$$
\operatorname{det}\left[B|A B| A^{2} B\right]=\alpha_{0}{ }^{2} \alpha_{1} k_{1}{ }^{2} k_{2} x_{1}^{* 2} x_{2}^{*} \neq 0,
$$

we get $\operatorname{rank}\left[B|A B| A^{2} B\right]=3$, and applying Theorem A.2 we obtain the local controllability of system (3.1)-(3.3) into $x^{*}$ on interval $[0, T]$.

The obtained local controllability means that from nearby states, the system can be steered into the equilibrium applying an appropriate small control $\bar{u} \in U_{\varepsilon_{0}}[0, T]$. Now we proceed to the determination of such a control.

Fix an initial state $x^{0}$ from a neighbourhood of local controllability of system (3.4), and for each control function $u$ small enough (i.e. $u \in U_{\varepsilon_{0}}[0, T]$, see conditions of system (A1)-(A2) of the Appendix), let $x$ be the solution of (3.4), defined on $[0, T]$ and corresponding to the initial value $x^{0}$. Then a control $u=\bar{u}$ will steer initial state $x^{0}$ into equilibrium $x^{*}$, if and only if it minimizes the functional

$$
\Phi(u):=\left|x(T)-x^{*}\right|^{2} .
$$

The above reasoning can be summarized in the following theorem:

Theorem 3.1. For any parameter values (2.1), system (3.1)-(3.3) is locally controllable into equilibrium $x^{*}$ on interval $[0, T]$, and an initial state $x^{0}$ will be steered 
1 into $x^{*}$ by a control $u \in U_{\varepsilon_{0}}[0, T]$ if and only if the latter is a solution of the following 2 optimal control problem:

$$
\begin{gathered}
\Phi(u):=\left|x(T)-x^{*}\right|^{2} \rightarrow \min , \\
u \in U_{\varepsilon_{0}}[0, T], x(0)=x^{0}, \\
\dot{x}=F\left(x, u^{*}+u(t)\right) .
\end{gathered}
$$

Remark 3.2. From the local controllability of the control system (3.4), we know that the optimal control problem (3.5)-(3.7) has at least one solution.

As a consequence of this theorem, for an effective calculation of an equilibrium control $\bar{u}$, it is enough to solve the optimal control problem (3.5)-(3.7). To this end we can apply the toolbox developed for MatLab in Banga, et al. (2005) and Hirmajer et al. (2009). Next, using this toolbox we will illustrate the results of Theorem 3.1.

Example 3.3. Let us consider system (3.1)-(3.3) with parameters of Example 2.3: $Q:=10 ; \alpha_{0}:=0.3 ; \alpha_{1}:=0.1 ; \beta_{1}:=0.2 ; \beta_{2}:=0.3 ; m_{1}:=0.1 ; m_{2}:=0.4 ; k_{1}:=0.5 ; k_{2}:=0.5$.

Taking as initial condition $x^{0}:=(4,7,5)$ and time interval $T:=5$, we apply the MatLab toolbox mentioned above. Figure $2 \mathrm{a}$ shows the solution $\bar{u}$ of the optimal control problem, the corresponding solution $x$, ending at equilibrium $x^{*}=(4.52,8,5.78)$ calculated in Example 2.3. can be seen in Figure 2b.

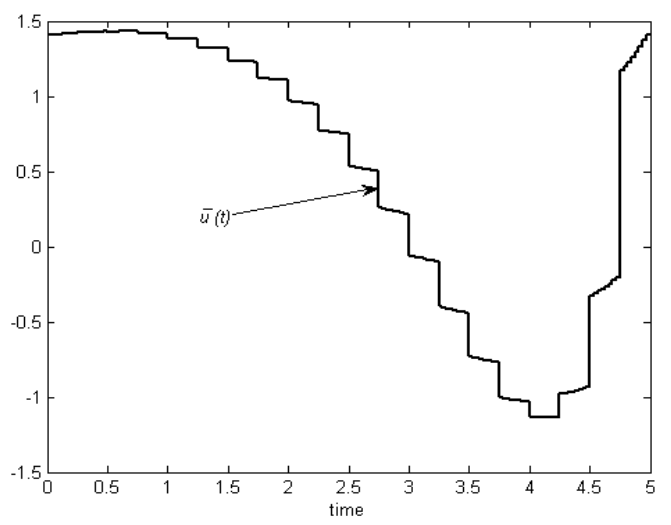

Figure 2a. Control function of system (3.1)-(3.3) for $T=5$, with initial value $x(0)=(4,7,5)$ 


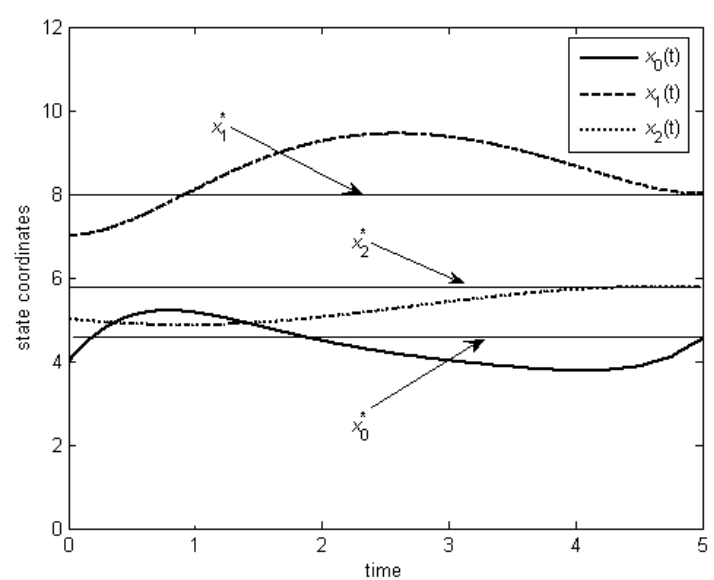

Figure 2b. Solutions of system (3.1)-(3.3) for $T=5$, with initial value $x(0)=(4,7,5)$

3 We note that, since by Remark 2.2, for the uncontrolled system, $x^{*}$ is asymptotically 4 stable, the state would tend to $x^{*}$, reaching it in "infinite time", as seen in Figure 2.c. 5 By our method the system state is steered into $x^{*}$ in given finite time.

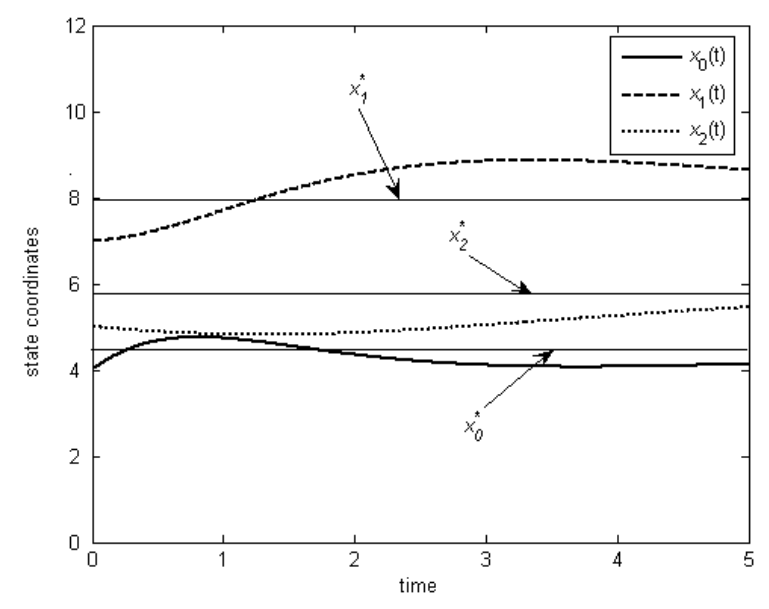

Figure 2c. Solution of system (2.2)-(2.4) for $T=5$, with initial value $x(0)=(4,7,5)$

7 Case 2. Control of a recycle rate

8 For the equilibrium control of the trophic chain, another possibility is to introduce 9 the control in one of the recycling rates, e.g. $\beta_{1}$. In this case the controlled system is of 10 the form

$$
\dot{x}_{0}=Q-\alpha_{0} x_{0} x_{1}+\left(\beta_{1}+u(t)\right) m_{1} x_{1}+\beta_{2} m_{2} x_{2}
$$


9 Now

$$
\begin{aligned}
& \dot{x}_{1}=x_{1}\left(-m_{1}+k_{1} \alpha_{0} x_{0}-\alpha_{1} x_{2}\right) \\
& \dot{x}_{2}=x_{2}\left(-m_{2}+k_{2} \alpha_{1} x_{1}\right) .
\end{aligned}
$$

Proceeding similarly to Case 1 , with definition

$$
F: \mathbf{R}^{4} \rightarrow \mathbf{R}^{3}, \quad F\left(x_{0}, x_{1}, x_{2}, u\right):=\left[\begin{array}{c}
Q-\alpha_{0} x_{0} x_{1}+\left(\beta_{1}+u\right) m_{1} x_{1}+\beta_{2} m_{2} x_{2} \\
x_{1}\left(-m_{1}+k_{1} \alpha_{0} x_{0}-\alpha_{1} x_{2}\right) \\
x_{2}\left(-m_{2}+k_{2} \alpha_{1} x_{1}\right)
\end{array}\right],
$$

control system (3.8)-(3.10) takes the form

$$
\dot{x}=F\left(x, u^{*}+u(t)\right) .
$$

The linearization process results in the same matrix $A$ as in Case 1, but in a different

$$
B=\left[\begin{array}{c}
m_{1} x_{1}^{*} \\
0 \\
0
\end{array}\right]
$$

$$
\operatorname{det}\left[B|A B| A^{2} B\right]=\alpha_{0}^{2} \alpha_{1} k_{1}^{2} k_{2} m_{1}^{3} x_{1}^{* 5} x_{2}^{*} \neq 0,
$$

therefore $\operatorname{rank}\left[B|A B| A^{2} B\right]=3$, and hence without any further assumption on the model parameters (2.1) Theorem A.2 implies local controllability of system (3.8)-(3.10) into $x^{*}$ on interval $[0, T]$.

\section{Case 3. Changing Malthus parameter into control variable}

For this case, as example, let us consider the control of the birth rate of the plant species. The obtained control system is

$$
\begin{aligned}
& \dot{x}_{0}=Q-\alpha_{0} x_{0} x_{1}+\beta_{1} m_{1} x_{1}+\beta_{2} m_{2} x_{2} \\
& \dot{x}_{1}=x_{1}\left(-m_{1}+u(t)+k_{1} \alpha_{0} x_{0}-\alpha_{1} x_{2}\right) \\
& \dot{x}_{2}=x_{2}\left(-m_{2}+k_{2} \alpha_{1} x_{1}\right) .
\end{aligned}
$$

Following the reasoning of the previous cases, matrix $A$ again is the same as in Case 1, whereas for $B$ we get

$$
B=\left[\begin{array}{c}
0 \\
x_{1}^{*} \\
0
\end{array}\right] \text {. }
$$

Since condition $\beta_{1} m_{1} \leq \alpha_{0} x_{0}{ }^{*}$ implies

$$
\operatorname{det}\left[B|A B| A^{2} B\right]=\alpha_{0} \alpha_{1} k_{2} x_{1}^{* 4} x_{2}^{*}\left(\alpha_{0} x_{0}^{*}-\beta_{1} m_{1}\right)+\beta_{2} m_{2} k_{2}^{2} \alpha_{1}^{2} x_{1}^{* 3} x_{2}^{* 2} \neq 0,
$$


1 and hence $\operatorname{rank}\left[B|A B| A^{2} B\right]=3$, applying again Theorem A.2 of the Appendix, we

2 obtain the following sufficient condition for the equilibrium control:

3 Theorem 3.4. If the recycling rate of the plant is small enough $\left(\beta_{1} \leq \frac{\alpha_{0} x_{0}{ }^{*}}{m_{1}}\right)$, then the trophic chain can be controlled to equilibrium, and an open-loop control can be found by solving an optimal control problem of the form (3.5)-(3.7).

\section{Closed-loop control to a new equilibrium}

Let us suppose that in an ecosystem over the past period an undesired stationary state has been formed. Then the objective of ecosystem management may be to control the system to a state where a given state component has a desired value, and keep it there in equilibrium, applying a constant control. Actually, in this way a controlled regime shift is realized.

To this end we will find a closed-loop (actually a linear feedback) control that asymptotically steers the system state. Unlike the open-loop control, where the intervention is calculated on beforehand, closed loop control means that at every moment the control to be applied is calculated from the current state of the system. For the construction we will follow the optimal control methodology of Rafikov et al. (2008), recalled in the Appendix.

The control of the trophic chain can be realized at different trophic levels. Below we present two possibilities: intervention either on the herbivore or on the plant, by adding or eliminating individuals of these populations.

\subsection{Steering the plant population to a given level by controlling the herbivore}

Let us suppose that we want to achieve a desired level $x_{1 d}^{*}$ of the plant species and keep it there in the long run, by means of changing the presence of the herbivore animal. Formally, let us consider the following control system

$$
\begin{aligned}
& \dot{x}_{0}=Q-\alpha_{0} x_{0} x_{1}+\beta_{1} m_{1} x_{1}+\beta_{2} m_{2} x_{2} \\
& \dot{x}_{1}=x_{1}\left(-m_{1}+k_{1} \alpha_{0} x_{0}-\alpha_{1} x_{2}\right) \\
& \dot{x}_{2}=x_{2}\left(-m_{2}+k_{2} \alpha_{1} x_{1}\right)+U
\end{aligned}
$$

where $U$ is a continuous control function. Given $x_{1 d}^{*}$, by solving system (4.2) below, we find an equilibrium $x_{d}^{*}=\left(x_{0 d}^{*}, x_{1 d}^{*}, x_{2 d}^{*}\right)$ and a corresponding constant control $u^{*} \in \mathbf{R}$ that would keep system (4.1) in equilibrium $x_{d}^{*}$ : 


$$
\begin{aligned}
& Q-\alpha_{0} x_{0 d}^{*} x_{1 d}^{*}+\beta_{1} m_{1} x_{1 d}^{*}+\beta_{2} m_{2} x_{2}^{*}=0 \\
& x_{1 d}^{*}\left(-m_{1}+k_{1} \alpha_{0} x_{0 d}^{*}-\alpha_{1} x_{2 d}^{*}\right)=0 \\
& x_{2 d}^{*}\left(-m_{2}+k_{2} \alpha_{1} x_{1 d}^{*}\right)+u^{*}=0
\end{aligned}
$$

Then, from (4.1) and (4.2), for the new variables,

$$
y=x-x_{d}^{*}, u=U-u^{*},
$$

we easily get the error system:

$$
\dot{y}=\hat{A} y+q(y)+\hat{B} u,
$$

where matrices $\hat{A}$ and $\hat{B}$ and the vector $q(y)$ are defined as follows:

$$
\hat{A}=\left[\begin{array}{ccc}
-\alpha_{0} x_{1 d}^{*} & -\alpha_{0} x_{0 d}^{*}+\beta_{1} m_{1} & \beta_{2} m_{2} \\
k_{1} \alpha_{0} x_{1 d}^{*} & 0 & -\alpha_{1} x_{1 d}^{*} \\
0 & k_{2} \alpha_{1} x_{2 d}^{*} & -m_{2}+k_{2} \alpha_{1} x_{1 d}^{*}
\end{array}\right], \hat{B}=\left[\begin{array}{l}
0 \\
0 \\
1
\end{array}\right], q(y)=\left[\begin{array}{c}
-\alpha_{0} y_{0} y_{1} \\
k_{1} \alpha_{0} y_{0} y_{1}-\alpha_{1} y_{1} y_{2} \\
k_{2} \alpha_{1} y_{1} y_{2}
\end{array}\right] .
$$

Now, in order to apply Theorem A.3 of Rafikov et al. (2008) (see Appendix), it is enough to find positive definite matrices $P, R, S \in \mathbf{R}^{3 \times 3}, P$ and $S$ symmetric, such that $P$ satisfies the matrix Riccati equation

$$
P \hat{A}+\hat{A}^{T} P-P \hat{B} R^{-1} \hat{B}^{T} P+S=0,
$$

and function

$$
l(y):=y^{T} S y-q^{T}(y) P y-y^{T} P q(y) \quad\left(y \in \mathbf{R}^{3}\right)
$$

is positive definite.

At this point we will need the following lemma.

Lemma 4.1. For any matrix $P \in \mathbf{R}^{3 \times 3}$ and

$$
S:=\left[\begin{array}{ccc}
1 & 0 & 0 \\
0 & 1 & 0 \\
0 & 0 & 1
\end{array}\right], \quad q(y):=\left[\begin{array}{c}
-\alpha_{0} y_{0} y_{1} \\
k_{1} \alpha_{0} y_{0} y_{1}-\alpha_{1} y_{1} y_{2} \\
k_{2} \alpha_{1} y_{1} y_{2}
\end{array}\right],
$$

function $l$ defined in (4.6) attains a strict local minimum at the origin $(0,0,0)$.

Proof. In the considered case, for all $\left(y \in \mathbf{R}^{3}\right)$ we have

$$
\begin{aligned}
l(y)= & y_{0}^{2}\left(1+\alpha_{0}\left(p_{11}+k_{1}\left(-p_{12}+p_{21}\right)\right) y_{1}\right)+y_{2}^{2}+\left(\alpha_{0} p_{13}+\alpha_{1}\left(p_{23}+p_{32}\right)\right) y_{1} y_{2}^{2}+ \\
& +y_{1}^{2}\left(1+\alpha_{0} p_{12} y_{2}+\alpha_{1}\left(2 p_{22}-k_{2}\left(p_{23}-p_{32}\right)\right) y_{2}\right)+ \\
& +y_{0} y_{1}\left(\alpha_{1}\left(p_{12}-k_{2}\left(p_{13}-p_{31}\right)+p_{21}\right) y_{2}+\alpha_{0}\left(p_{21} y_{1}+\left(p_{11}+k_{1}\left(p_{23}-p_{32}\right)+p_{31}\right) y_{2}\right)\right),
\end{aligned}
$$

and its first order partial derivatives are

$$
D_{0} l(y)=2 y_{0}\left(1+\alpha_{0}\left(p_{11}-k_{1}\left(p_{12}-p_{21}\right)\right) y_{1}\right)+y_{1}\left(\alpha_{1}\left(p_{12}-k_{2}\left(p_{13}-p_{31}\right)+p_{21}\right) y_{2}+\right.
$$




$$
\begin{aligned}
& \left.+\alpha_{0}\left(p_{21} y_{1}+\left(p_{11}+k_{1}\left(p_{23}-p_{32}\right)+p_{31}\right) y_{2}\right)\right) \\
D_{1} l(y)= & \alpha_{0}\left(p_{11}+k_{1}\left(-p_{12}+p_{21}\right)\right) y_{0}^{2}+\alpha_{0} p_{21} y_{0} p_{1}+\left(\alpha_{0} p_{13}+\alpha_{1}\left(p_{23}+p_{32}\right)\right) y_{2}^{2}+ \\
+ & \left.2 y_{1}\left(1+\alpha_{0} p_{12} y_{2}+\alpha_{1}\left(2 p_{22}-k_{2} p_{23}-p_{32}\right)\right) y_{2}\right)+y_{0}\left(\alpha_{1}\left(p_{12}-k_{2}\left(p_{13}-p_{31}\right)+p_{21}\right) y_{2}+\right. \\
& +\alpha_{0}\left(p_{21} y_{1}+\left(p_{11}+k_{1}\left(p_{23}-p_{32}\right)+p_{31}\right) y_{2}\right) \\
D_{2} l(y)= & \left(\alpha_{1}\left(p_{12}+p_{21}-k_{2}\left(p_{13}-p_{31}\right)\right)+\alpha_{0}\left(p_{11}+k_{1}\left(p_{23}-p_{32}\right)\right)\right) y_{0} y_{1}+ \\
& \left(\alpha_{0} p_{12}+\alpha_{1}\left(2 p_{22}+k_{2}\left(-p_{23}+p_{32}\right)\right)\right) y_{1}^{2}+2 y_{2}+2\left(\alpha_{0} p_{13}+\alpha_{1}\left(p_{23}+p_{32}\right)\right) y_{1} y_{2} .
\end{aligned}
$$

Obviously

$$
D_{0} l(0)=D_{1} l(0)=D_{2} l(0)=0,
$$

and for the Hessian of $l$ at the origin we have

$$
H l(0)=\left[\begin{array}{lll}
2 & 0 & 0 \\
0 & 2 & 0 \\
0 & 0 & 2
\end{array}\right],
$$

which is obviously positive definite, implying that the origin is a strict local minimum point of function $l$

Now, from the above reasoning, combining Theorem A.3 with Corollary A.6 of Appendix, we obtain the following theorem.

Theorem 4.2. Suppose that there exist positive definite matrices $P, R \in \mathbf{R}^{3 \times 3}, P$ symmetric, such that with matrices $\hat{A}, \hat{B}$ defined in (4.4), $P$ is a solution of the matrix Riccati equation (4.5). Then the linear feedback

$$
u(y):=-R^{-1} \hat{B}^{T} P y \quad\left(y \in \mathbf{R}^{3}\right)
$$

asymptotically steers any initial state $y(0)$ into zero. In particular, there exists a neighbourhood $V$ of zero in $\mathbf{R}^{3}$ such that for all initial value $x(0) \in V$ and control $U=u^{*}+u$, for the solution $x$ of system (4.1) we have $\lim _{\infty} x=x_{d}^{*}$.

Let us consider now an illustrative example.

Example 4.3. Let us start from the uncontrolled system (2.2)-(2.4) with the same model parameters as in Example 2.3. As we have seen, system (2.2)-(2.4) has a positive equilibrium $x^{*}=(4.52,8,5.78)$, which is asymptotically stable.

Let us assume that we want to steer the biomass level to a desired level $x_{1 d}^{*}=4$, intervening on the herbivore animal. From system of algebraic equations (4.2), we calculate $x_{0 d}^{*}, x_{2 d}^{*}$ and $u^{*}$, obtaining $x_{d}^{*}=(9.76,4,13.65), u^{*}=2.73$. 
1 For matrices $\hat{A}$ and $\hat{B}$ defined in (4.4) we have

$$
\hat{A}=\left[\begin{array}{ccc}
-1.2 & -2.91 & 0.12 \\
0.6 & 0 & -0.4 \\
0 & 0.68 & -0.2
\end{array}\right] ; \hat{B}=\left[\begin{array}{l}
0 \\
0 \\
1
\end{array}\right]
$$

3 and choosing

$$
S:=\left[\begin{array}{lll}
1 & 0 & 0 \\
0 & 1 & 0 \\
0 & 0 & 1
\end{array}\right], \quad R:=[1],
$$

5 we calculate matrix $P$ from the Riccati equation (4.5) using the Matlab 7.6 command $6 \quad$ LQR, obtaining

$$
P=\left[\begin{array}{ccc}
0.47 & 0.12 & -0.05 \\
0.12 & 2.59 & -0.23 \\
-0.05 & -0.23 & 0.9
\end{array}\right]
$$

8 Obviously $P$ and $Q$ are positive definite symmetric matrices. Now, for the auxiliary 10 function $l$ we obtain

$$
l(y)=y_{0}^{2}\left(1+0.14 y_{1}\right)+y_{0} y_{1}\left(0.36 y_{1}+0.19 y_{2}\right)+y_{1}^{2}\left(1+0.55 y_{2}\right)+y_{2}^{2}-0.061 y_{1} y_{2}^{2},
$$
and its local definiteness is illustrated in Figure 3.

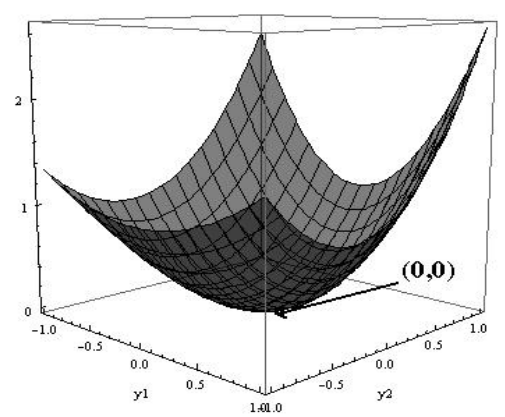

Section $y_{0}=0$

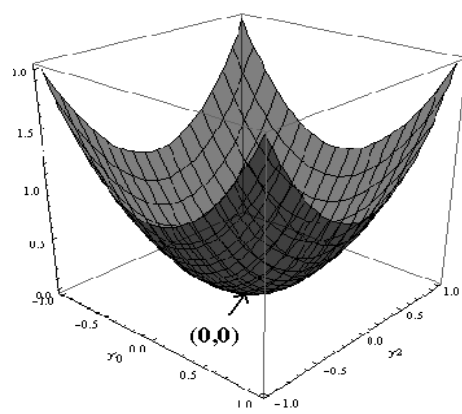

Section $y_{1}=0$

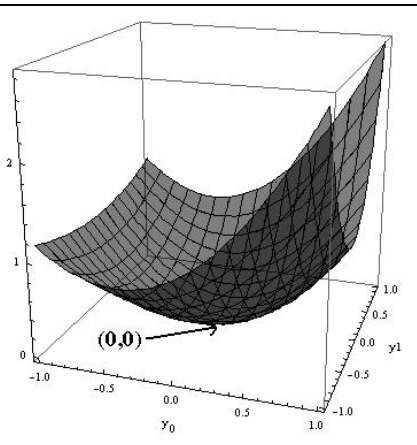

Section $y_{2}=0$

Figure 3. 3D sections of $l$ at the origin

Applying Theorem 4.2, the feedback control

$$
u(y)=0.05 y_{0}+0.23 y_{1}-0.9 y_{2}
$$

asymptotically steers the error system (4.3) to 0 , implying that for the solution $x$ of system (4.1) with the closed-loop control $U(x):=u^{*}+u\left(x-x_{d}^{*}\right)$, we have $\lim _{\infty} x=x_{d}^{*}=(9.76,4,13.65)$, as shown in Figure 4. 


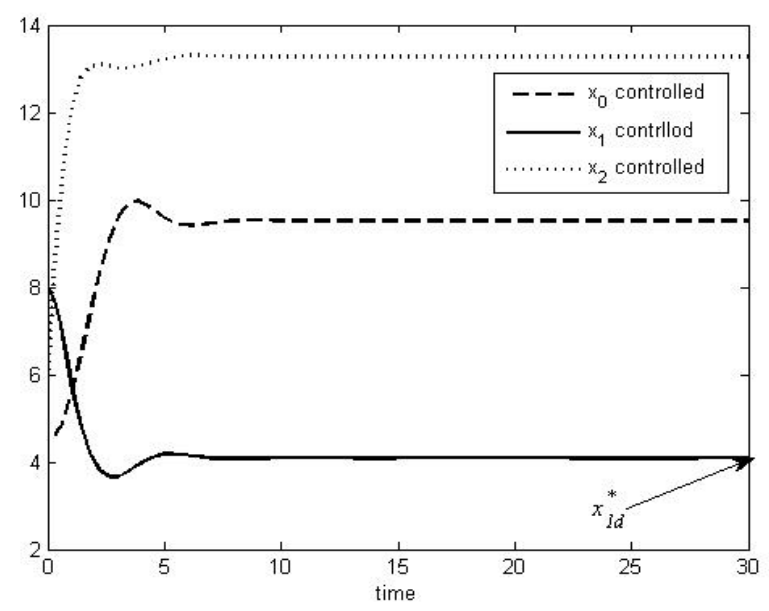

Figure 4. Solution of system (4.1), with parameters of Example 2.3, and initial value $x(0)=x^{*}=(4.52,8,5.78)$

\subsection{Steering the animal population by controlling the plant}

Let us consider now the problem which in certain sense is the opposite to the previous one. We want to achieve a desired level $x_{2 d}^{*}$ of the animal population and keep it there in a stationary regime, means of changing the presence of the plant species.

The corresponding control system then is

$$
\begin{aligned}
& \dot{x}_{0}=Q-\alpha_{0} x_{0} x_{1}+\beta_{1} m_{1} x_{1}+\beta_{2} m_{2} x_{2} \\
& \dot{x}_{1}=x_{1}\left(-m_{1}+k_{1} \alpha_{0} x_{0}-\alpha_{1} x_{2}\right)+U \\
& \dot{x}_{2}=x_{2}\left(-m_{2}+k_{2} \alpha_{1} x_{1}\right)
\end{aligned}
$$

where $U$ is again, a continuous control function. We suppose that to a constant control $u^{*} \in R$, there corresponds a desired state $x_{d}^{*}$, i.e.,

$$
\begin{aligned}
& Q-\alpha_{0} x_{0 d}^{*} x_{1 d}^{*}+\beta_{1} m_{1} x_{1 d}^{*}+\beta_{2} m_{2} x_{2 d}^{*}=0 \\
& x_{1 d}^{*}\left(-m_{1}+k_{1} \alpha_{0} x_{0 d}^{*}-\alpha_{1} x_{2 d}^{*}\right)+u^{*}=0 \\
& x_{2 d}^{*}\left(-m_{2}+k_{2} \alpha_{1} x_{1 d}^{*}\right)=0
\end{aligned}
$$

Reasoning in a way analogous to the previous case, applying Theorem 4.2 we can find an appropriate control that steers the system into the desired new equilibrium state, which can be seen in the following illustrative example.

Example 4.4. We consider the same model parameters of Examples 2.3 and 4.3 that imply the positive asymptotically stable equilibrium $x^{*}=(4.52,8,5.78)$. Now, we intervene on the plant species in order to steer the herbivore population to a higher level, 
$1 \quad x_{2 d}^{*}=10$. From system (4.9) we calculate the constant control $u^{*}$, and the rest of

2 coordinates corresponding to the desired state, obtaining $x_{d}^{*}=(4.73,8,10) ; u^{*}=3.12$.

3 Matrices $\hat{A}$ and $\hat{B}$ in this case are

$$
\hat{A}=\left[\begin{array}{ccc}
-2.4 & -1.4 & 0.12 \\
1.2 & 0 & -0.8 \\
0 & 0.5 & 0
\end{array}\right] ; \hat{B}=\left[\begin{array}{l}
0 \\
1 \\
0
\end{array}\right]
$$

5 Choosing $S$ and $R$ the same as in Example 4.3 and calculating matrix $P$ from the

6 corresponding Riccati equation we obtain

$$
P=\left[\begin{array}{lll}
0.31 & 0.22 & 0.14 \\
0.22 & 0.93 & 0.49 \\
0.14 & 0.49 & 2.74
\end{array}\right]
$$

8 Now, from formula (4.7) the linear feedback for the error system is

$$
u(\mathrm{y})=-0.22 y_{0}-0.93 y_{1}-0.49 y_{2},
$$

and hence, for system (4.8) the required closed-loop $U(x):=u^{*}+u\left(x-x_{d}^{*}\right)$ can be

11 obtained. The resulting solution of the controlled system can be seen in Figure 5.

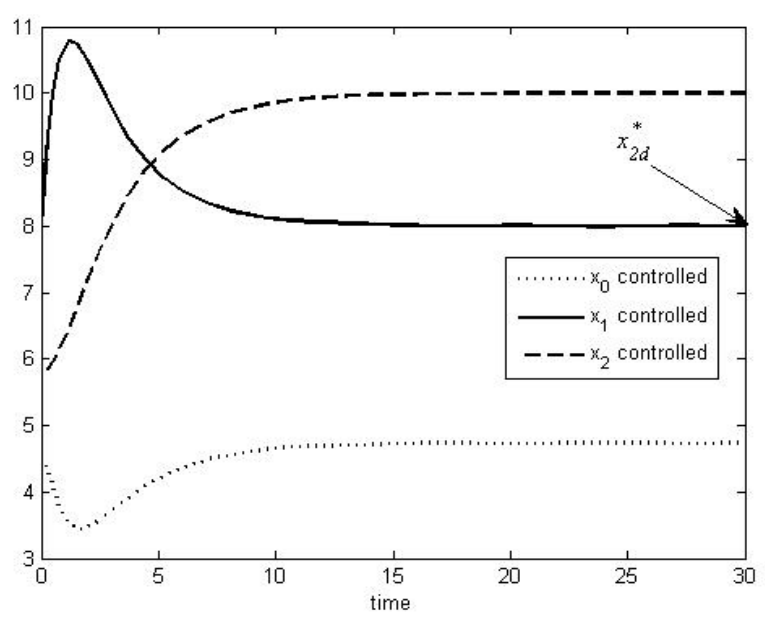

Figure 5. Solution of system (4.8) with parameters of Example 2.3, and initial value $x(0)=x^{*}=(4.52,8,5.78)$

\section{Discussion}

In the paper a control-theoretic methodology has been proposed for a particular tasks of ecosystem management. We have shown how the technique of optimal control theory can be applied to deal with qualitative properties like open- or closed-loop equilibrium control of ecological systems. For the different tasks we used different 
1 approaches: the constructed open-loop control substitutes certain model parameter,

while the closed-loop control necessarily acts on the state variables.

In Petrosjan and Zakharov (1997) the controllability of predator-prey was already considered, but only with constant control. In Varga (2008b), sufficient condition it was obtained for a Lotka-Volterra system to locally controllable into equilibrium, but no method was proposed to calculate the existing equilibrium control. Concerning the open-loop equilibrium control, the novelty of our paper is that for the considered trophic chain, an efficient method for the calculation of the equilibrium control is proposed, by setting up and solving an optimal control problem.

In Rafikov et al. (2008), an optimal feedback control was used in order to steer a Lotka-Volterra type predator-prey system asymptotically into a given equilibrium, in the context of biological pest control. We have shown, instead, that a similar approach can be also applied to find a closed-loop equilibrium control of a non-Lotka-Volterra type trophic chain into a new equilibrium, realizing a controlled regime shift.

In the present paper the proposed methodology has been used for the control of simple trophic chain. However, it can be also applied to different types of multispecies dynamic population models, see e.g. Yodzis (1989), Cressman et al. (2001), Cressman and Garay (2003), (2006), (2009), Garay et al. (2003), Cressman et al. (2004), Garay (2002), (2009).

\section{Acknowledgements}

This paper has been realized in the framework of the Hungarian-Spanish intergovernmental scientific and technological collaboration, with the support of the Scientific and Technological Innovation Fund (of Hungary, ES-17/2008), the Ministry of Education and Sciences (of Spain, HH2008-0023) and the Hungarian Scientific Research Fund OTKA K 68187, K 81279, K 62000. The authors are grateful to the amonymous referees for the valuables comments.

\section{References}

Banga, J. R., Balsa-Canto, E., Moles, C. G. and Alonso, A. A., 2005. Dynamic optimization of bioprocesses: Efficient and robust numerical strategies. Journal of Biothecnology, 117, 407-419.

Chen, Ben M.; Lin, Zongli; Shamesh, Yacov A., 2004. Linear Systems Theory. A Structural Decomposition Approach. Birkhauser, Boston.

Cressman, R., Garay, J., 2003. Stablility $N$-species coevolutionary systems. Theoretical Population Biology, 64, 519-533.

Cressman, R., Garay, J., 2006. A game-theoretical model for punctuated equilibrium: species invasion and stasis through coevolution. BioSystems, 84, 1-14. 
Cressman, R., Garay, J., 2009. A Predator-Prey Refuge System: Evolutionary Stability in Ecological Systems. Theoretical Populations Biology, 76, 248-257.

Cressman, R., Garay, J., Hofbauer, J., 2001. Evolutionary stability concepts for $N$ species frequency-dependent interactions. Journal of Theoretical Biology, 211, 1-10.

Cressman, R., Krivan, V., Garay, J., 2004. Ideal free distributions, evolutionary games, and population dynamics in multiple-species environments. American Naturalist, 164, No. 4, 473-489.

Fath, Brian D., 2004. Distributed control in ecological networks. Ecological Modelling, $179,235-245$.

Gámez, M.; López, I. and Molnár, S., 2008. Monitoring environmental change in an ecosystem. Biosystems, 93, 211-217.

Gámez, M., López, I., Garay, J. and Z. Varga, 2009. Observation and control in a model of a cell population affected by radiation. BioSystems 96, 172-177.

Garay, J., 2002. Many Species Partial Adaptive Dynamics. BioSystems, 65, 19-23.

Garay, J., 2009. Cooperation in defence against predator. Journal of Theoretical Biology 257, 45-51.

Garay, J., Cressman, R., Varga, Z., 2003. Optimal behaviour of honey bees based on imitation at fixed densities. Community Ecology, 4 No. 2, 219-224.

Hirmajer, T., Balsa-Canto, E. and Banga, J. R., 2009. DOTcvpSB, a software toolbox for dynamic optimization in systems biology. BMC Bioinformatics, 10:199.

Jorgensen, S., Svirezhev, Y. (Eds.), 2004. Towards a Thermodynamic Theory for Ecological Systems, Pergamon.

Kalman, R. E., Falb, P. L., Arbib, M. A., 1969. Topics in Mathematical System Theory. McGraw-Hill, New York.

Kósa, A., Varga, Z., 1996. Controllability of evolutionary game dynamics. Bulletin of the University of Agricultural Sciences, Gödöllö, Vol. 75 (II), 43-49.

Lee, E.B. and Markus, L., 1971. Foundations of Optimal Control Theory. New YorkLondon-Sydney, Wiley.

López I, Gámez, M., Carreño, R. (2004), Observability in dynamic evolutionary models. BioSystems, 73 No. 2, 99-109.

López I, Gámez M, Molnár, S., 2007. Observability and observers in a food web. Applied Mathematics Letters, 20 No. 8, 951-957.

Molnár, S., 1987. Model runs for the definition of the most advantageous integrated energetical verticum in the national economy, Publications of Central Mining Development Institute, 30, 121-127.

Molnár, S., 1988a. Realization of verticum-type systems, Mathematical Analysis and Systems Theory 5, 11-30. Karl Marx University of Economics, Budapest.

Molnár, S., 1988b. Optimization of realization-independent cost functions, Mathematical Analysis and Systems Theory 5, 1-10. Department of Mathematics, Karl Marx University of Economics, Budapest.

Molnár, S., 1988c. Observability and controllability of decomposed systems I, Mathematical Analysis and Systems Theory 5, 57-66. Department of Mathematics, Karl Marx, University of Economics, Budapest.

Molnár, S., 1988d. Observability and controllability of decomposed systems II, Mathematical Analysis and Systems Theory 5, 67-72. Department of Mathematics, Karl Marx University of Economics, Budapest.

Molnár, S., 1988e. Observability and controllability of decomposed systems III, Mathematical Analysis and Systems Theory 5, 73 -80. Department of Mathematics, Karl Marx University of Economics, Budapest. 
Molnár, S., 1989. A special decomposition of linear systems, Belgian Journal Operations Reseach, Statistics and. Computation Science, 29 No. 4, 1 -19.

Molnár, S., 1993. Stabilization of verticum-type systems, Pure Mathematics and Applications 4 No. 4, $493-499$.

Molnár, S., Szigeti, F., 1994. On verticum-type linear systems with time-dependent linkage, Applied Mathematics and Computation, 60, 89-102.

Petrosjan, L. A. and Zakharov, V.V., 1997. Mathematical Models in Environmental Policy Analysis. Nova Science Publishers, Inc. N.Y. USA

Rafikov, M., Balthazar, J.M., Bremen, H.F. von, 2008. Mathematical Modelling and Control of Population Systems: Applications in Biological Pest Control. Applied Mathematics and Computation, 2008, No. 2, 557-573.

Scarelli, A., Varga, Z., 2002. Controllability of selection-mutation systems. Biosystems, 65 No 2-3, 113-121.

Shamandy, A., 2005. Monitoring of trophic chains. Biosystems, Vol. 81, Issue 1, 43-48.

Svirezhev, Yu.M. and D.O. Logofet, 1983. Stability of biological communities. Mir Publishers, Moscow.

Varga, Z., 1989. On controllability of Fisher's model of selection. In: Dafermos, C.M.,Ladas, G., Papanicolaou (eds), Differential Equations. Marcel Dekker, New York (1989) 717-723.

Varga, Z., 1990. Control-theoretical problems in population genetics. Biometric Bulletin Vol. 7 No 1, p. 11.

Varga, Z., 1992. On Observability of Fisher's model of selection. Pure Mathematics and Applications, Ser. B. Vol. 3 No 1, 15-25.

Varga, Z., Scarelli, A. and Shamandy, A., 2003. State monitoring of a population system in changing environment. Community Ecology 4 No. 1, 73-78.

Varga, Z., 2008a. Observer Design for Genetic Processes. Mechanical Engineering Letters, Vol. 1, 13-25.

Varga, Z., 2008b. Applications of mathematical systems theory in population biology. Periodica Mathematica Hungarica, Vol. 56 No. 1, 157-168.

Yodzis, P., 1989. Introduction to Theoretical Ecology. Harper \& Row. New York.

\section{Appendix}

Controllability of nonlinear systems

First, from Lee and Markus (1971), we recall some concepts and a theorem of nonlinear control theory. Given $m, s \in \mathbf{N}$, let $F: \mathbf{R}^{m} \times \mathbf{R}^{s} \rightarrow \mathbf{R}^{m}$ be a continuously differentiable function. For a reference control value $u^{*} \in \mathbf{R}^{s}$, let $x^{*} \in \mathbf{R}^{m}$ be such that $F\left(x^{*}, u^{*}\right)=0$. For technical reason we shall need a rather general class of controls. Let us fix a time interval $[0, T]$, and for each $\varepsilon \in \mathbf{R}^{+}$define the class of essentially bounded $\varepsilon$ - controls

$$
U_{\varepsilon}[0, T]:=\left\{u \in L_{\infty}^{s}[0, T] \mid\|u(t)\|_{\infty} \leq \varepsilon \text { for almost every } t \in[0, T]\right\} .
$$

Then it can be shown that there exists $\varepsilon_{0} \in \mathbf{R}^{+}$such that for all $u \in U_{\varepsilon_{0}}[0, T]$ and $x^{0} \in \mathbf{R}^{m}$ with $\left\|x^{0}-x^{*}\right\|<\varepsilon_{0}$ the initial value problem 


$$
\begin{aligned}
& \dot{x}(t)=F\left(x(t), u^{*}+u(t)\right) \quad(\text { for a.e. } t \in[0, T]) \\
& x(0)=x^{0}
\end{aligned}
$$

has a unique solution. We notice that $x^{*}$ is an equilibrium state for the zero-control system.

Definition A.1. Control system (A.1)-(A.2) is said to be locally controllable to $x^{*}$ on $[0, T]$, if there exists $\left.\varepsilon \in] 0, \varepsilon_{0}\right]$ such that for all $x^{0}$ from the $\varepsilon$-neighbourhood of $x^{*}$, there is a control $u \in U_{\varepsilon}[0, T]$ that controls the initial state $x^{0}$ to equilibrium $x^{*}$, i.e. for the solution $x$ of the initial value problem (A.1)-(A.2), equality $x(T)=x^{*}$ holds. Let us linearize system (A.1)-(A.2) around $\left(x^{*}, u^{*}\right)$, introducing the corresponding Jacobians

$$
A:=D_{1} F\left(x^{*}, u^{*}\right), \quad B:=D_{2} F\left(x^{*}, u^{*}\right) .
$$

Then we have the following sufficient condition for local controllability:

Theorem A.2 (Lee and Markus, 1971)

If $\left.\operatorname{rang}|B| A B|..| A^{n-1} B\right]=n$ then system (A.1)-(A.2) is locally controllable to $x^{*}$ on $[0, T]$.

\section{Closed-loop asymptotic control into equilibrium in nonlinear systems}

Now, from Rafikov et al. (2008) we recall the construction of a linear closed-loop control that asymptotically steers the system into a desired equilibrium. For given $n, r \in \mathbf{N}, \hat{A} \in \mathbf{R}^{n x n}, \hat{B} \in \mathbf{R}^{n \times r}$ and continuously differentiable function $g: \mathbf{R}^{n} \rightarrow \mathbf{R}^{n}$, consider the control system

$$
\dot{x}=\hat{A} x+g(x)+\hat{B} U,
$$

where $U$ is a continuous control function. Assume that to a constant control $u^{*} \in \mathbf{R}^{r}$, there corresponds an equilibrium state $x^{*}$, i.e.,

$$
\hat{A} x^{*}+g\left(x^{*}\right)+\hat{B} u^{*}=0 .
$$

Then, from (A.3) and (A.4), with substitutions

$$
y:=x-x^{*} ; u:=U-u^{*}
$$

we obtain

$$
\dot{y}=\hat{A} y+q(y)+\hat{B} u, \quad \text { where } \quad q(y):=g\left(y+x^{*}\right)-g\left(x^{*}\right) .
$$

Below a feedback control will be given which asymptotically steers system (A.5) into the zero equilibrium. 
Theorem A.3 (Rafikov et al. 2008). If there exist positive definite matrices

$2 P, R, S \in \mathbf{R}^{n \times n}, P$ and $S$ symmetric, such that the function

$$
l(y):=y^{T} S y-q^{T}(y) P y-y^{T} P q(y) \quad\left(y \in \mathbf{R}^{n}\right)
$$

4 is positive definite, and $P$ satisfies the equation

5

$$
P \hat{A}+\hat{A}^{T} P-P \hat{B} R^{-1} \hat{B}^{T} P+S=0 .
$$

6 Then the linear feedback

$$
u(y)=-R^{-1} \hat{B}^{T} P y \quad\left(y \in \mathbf{R}^{n}\right)
$$

8 asymptotically steers any initial state $y(0)$ into zero.

Remark A.4. The statement $\lim _{\infty} y=0$ is obviously equivalent to $\lim _{\infty} x=x^{*}$.

Remark A.5. According to Rafikov et al. 2008, the feedback control (A.6) also 11 minimizes the functional

$$
I(y):=\int_{0}^{\infty}\left[l(y(t))+u^{T}(y(t)) R u(y(t))\right] d t,
$$

13 however, we will not use this statement.

14 Corollary A.6. (Gámez et al. 2009) Using the notation of the previous Theorem 15 A.3, let us suppose that function $l$ is locally positive definite. Then there exists a 16 neighbourhood $V$ of zero in $\mathbf{R}^{n}$ such that for all initial value $x(0) \in V$, for the solution $x$ 17 of system (A.6) we have $\lim _{\infty} x=x^{*}$. 